\title{
Pararenal Leiomyosarcoma of the Inferior Vena Cava
}

\begin{abstract}
A complete surgical resection is the only proven therapeutic modality that prolongs the survival in patients with leiomyosarcoma of the inferior vena cava (IVC). Reconstruction of the IVC is not always necessary but is often required to facilitate venous drainage of the kidney for the tumors at the pararenal area of the IVC. Controversy exists in postoperative adjuvant therapy. Recently, we experienced four cases of pararenal leiomyosarcoma of the IVC, of which treatment consisted of a complete resection of the tumor, ringed polytetrafluoroethylene (PTFE) graft interposition, and bilateral renal vein reconstructions in all patients. Postoperative radiation therapy was instituted in 3 of 4 patients. One patient who did not receive the postoperative radiation therapy was treated with adjuvant chemotherapy. The kidneys were preserved in all patients and no deep vein thrombosis (DVT) or venous insufficiency of the lower extremity veins developed. Distant metastasis to the lung was noted in one patient at 18 months after surgery, who was not received the postoperative radiation therapy but chemotherapy. In conclusion, a complete resection of the tumor, IVC reconstruction, and bilateral renal vein reconstruction followed by adjuvant radiation therapy is recommended for the treatment of pararenal leiomyosarcoma of the IVC.
\end{abstract}

Key Words : Leiomyosarcoma; Vena Cava, Inferior; Retroperitoneal Neoplasms

\author{
Tae-Won Kwon, KyurBo Sung*, \\ Yong-FI Cho, Do-Kyun Km, Sun-Mo Yang, \\ Jae-Yoon $\mathrm{Bo}^{\dagger}$, Geun-Eun Kim
}

Division of Vascular Surgery, Department of Surgery, Department of Radiology ${ }^{*}$, and Department of Pathology ${ }^{\ddagger}$ University of Ulsan College of Medicine and Asan Medical Center, Seoul, Korea

Received : 6 January 2003

Accepted : 7 February 2003

Address for correspondenœ

Tae-Won Kwon, M.D.

Division of Vascular Surgery, Department of Surgery, University of Ulsan College of Medicine and Asan Medical Center, 388-1 Pungnap-2dong, Songpa-gu, Seoul 138-736, Korea

Tel : +82.2-3010-3492, Fax : +82.2-474-9027

E-mail : twkwon2@amc.seoul.kr

*We have no financial support or commercial associations that might pose a conflict of interest in connection with the submitted manuscript.

\section{INTRODUCTION}

Leiomyosarcoma of the inferior vena cava (IVC) is a rare malignant tumor originating from the smooth muscle fibers of the media. Unlike intravenous leiomyomatosis which usually occurs in the veins of the myometrium and shows well differentiated smooth muscle cells with no or only a modest degree of nuclear pleomorphism and no or rare mitotic figures, leiomyosarcoma arises preferentially in the vena cava and usually shows high-grade tumors with easily recognized mitotic figures. Although only 218 cases of leiomyosarcoma of IVC have been reported in the international registry until 1994, leiomyosarcoma is the most common malignant tumor of the IVC (1). A complete resection of the tumor is the only treatment modality which may guarantee a long-term disease-free survival (2). When it comes to pararenal leiomyosarcoma of the IVC, however, the IVC and the renal vein reconstruction following resection of the tumor is necessary to prevent transient or permanent renal dysfunction. We herein report four additional cases of leiomyosarcoma of the IVC, emphasizing the surgical procedure and possible benefit of postoperative adjuvant radiotherapy.

\section{MATERIALS AND METHODS}

Between April 1999 and May 2002, the authors treated four patients with pararenal leiomyosarcoma of the IVC. We analyzed the clinicopathologic findings of these patients retrospectively.

\section{Patients}

All of the patients were referred to our hospital under the diagnosis of the retroperitoneal sarcoma on an abdominal computerized tomography (CT) scan (Fig. 1). Transfemoral caval biopsy was performed and the diagnosis of leiomyosarcoma of the IVC was made in one patient preoperatively. A laparotomy had been performed only for biopsy in one patient. All patients were female and the mean age was $50.8 \mathrm{yr}$ (range 27 to $63 \mathrm{yr}$ ). All patients complained of upper abdominal pain for 12 to 36 months. An abdominal mass was palpated on physical examination in one patient. Past medical history was noncontributory in all of the patients. Preoperative laboratory or imaging studies showed no other abnormal findings or no evidence of distant metastasis in all patients.

\section{Operation}

In every case, we used an inverted $\mathrm{T}$ skin incision, mobilized the right and caudate lobes of the liver from the IVC, and prepared proximal and distal vascular control of the IVC at about $2-3 \mathrm{~cm}$ from the macroscopic tumor margins. Both the right and the left main renal veins were dissected. After 

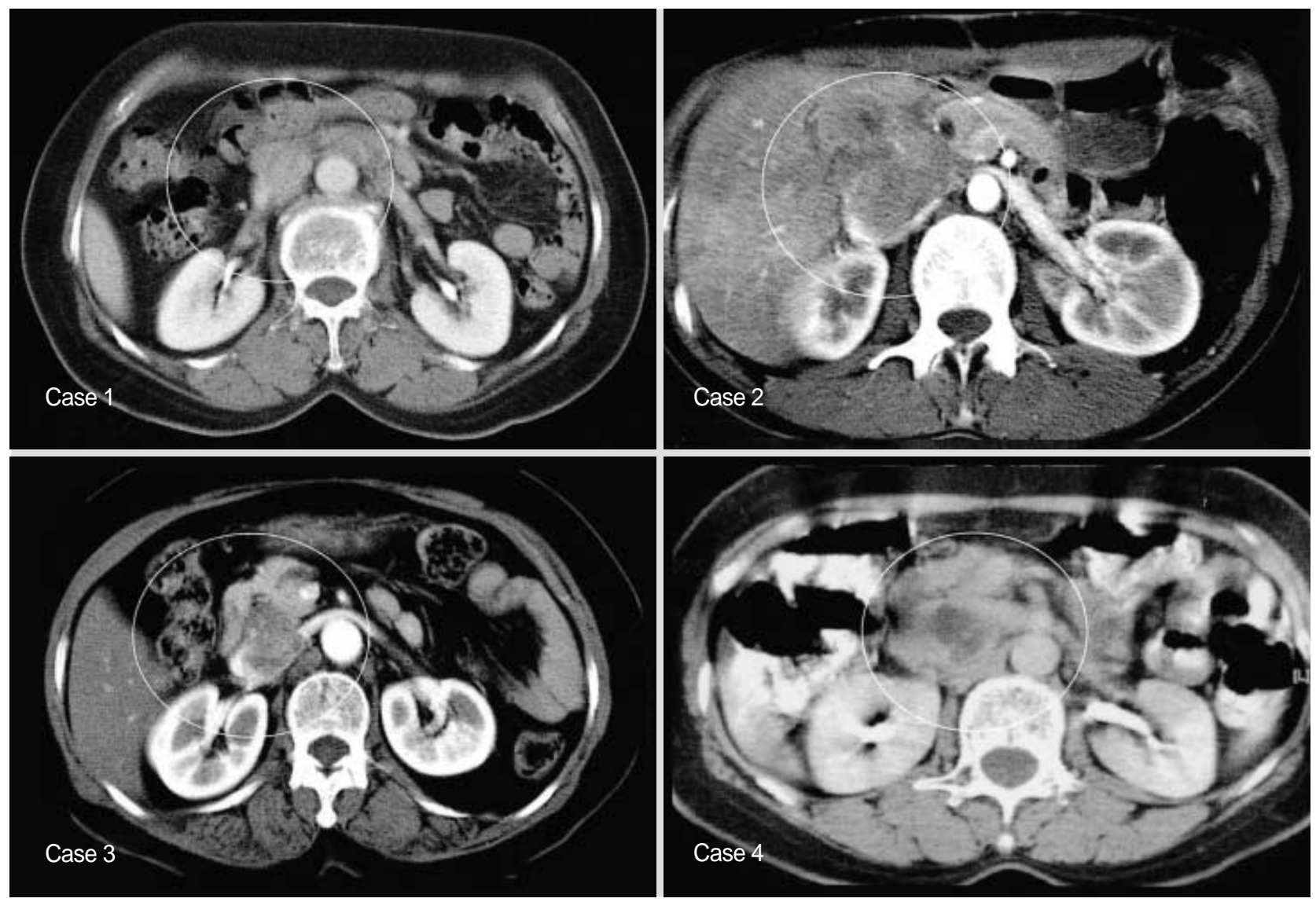

Fig. 1. Preoperative abdominal computerized tomography (CT) scan of four patients. Pararenal leiomyosarcoma of the inferior vena cava (white circle) are seen on an abdominal CT scan in all four patients.

systemic heparinization, a proximal IVC clamping was performed step by step with monitoring the systemic blood pressure. Then, both renal veins and distal IVC were clamped. We routinely performed ringed polytetrafluoroethylene (PTFE) graft interposition and bilateral renal vein reconstructions; direct reimplantation of the right renal vein to the graft in all four patients, PTFE graft interposition between the left renal vein and the graft in three patients, and left renal vein ligation in one patient, following a complete resection of the tumor (Fig. 2). There was no systemic hypotension or circulatory collapse during the procedure in all patients. Some degree of renal congestion, especially in the right kidney, was noted intraoperatively but completely relieved on declamping in all cases. As a result, all kidneys were preserved and no postoperative renal functional impairment was noted (Fig. 3). Venovenous bypass and extracorporeal circulation techniques were not used in any of our cases.

\section{Adjuvant therapy}

All patients received postoperative adjuvant radiotherapy and/or chemotherapy. Radiotherapy at a does of 45-55 Gy was performed in three patients. Six cycles of chemotherapy (cyclophosphamide, doxorubicine, and dacarbazine) was given to two patients. One patient received both chemotherapy and radiotherapy.

\section{RESULTS}

\section{Postoperative course}

Postoperative course was uneventful in all patients except one, who required a second laparotomy for bleeding control on the first postoperative day. Kidneys were preserved in all patients and no deep vein thrombosis (DVT) or venous insufficiency of the lower extremity veins developed during the 6-41 months of follow-up period.

\section{Pathology}

All of the patients were considered to have a curative resection intraoperatively. One patient, however, showed a positive resection margin pathologically. The other patients had free tumor margins. The tumors ranged from $5 \mathrm{~cm}$ to $8.9 \mathrm{~cm}$ in greatest dimension (average, $7.05 \mathrm{~cm}$ ) and formed nodular 

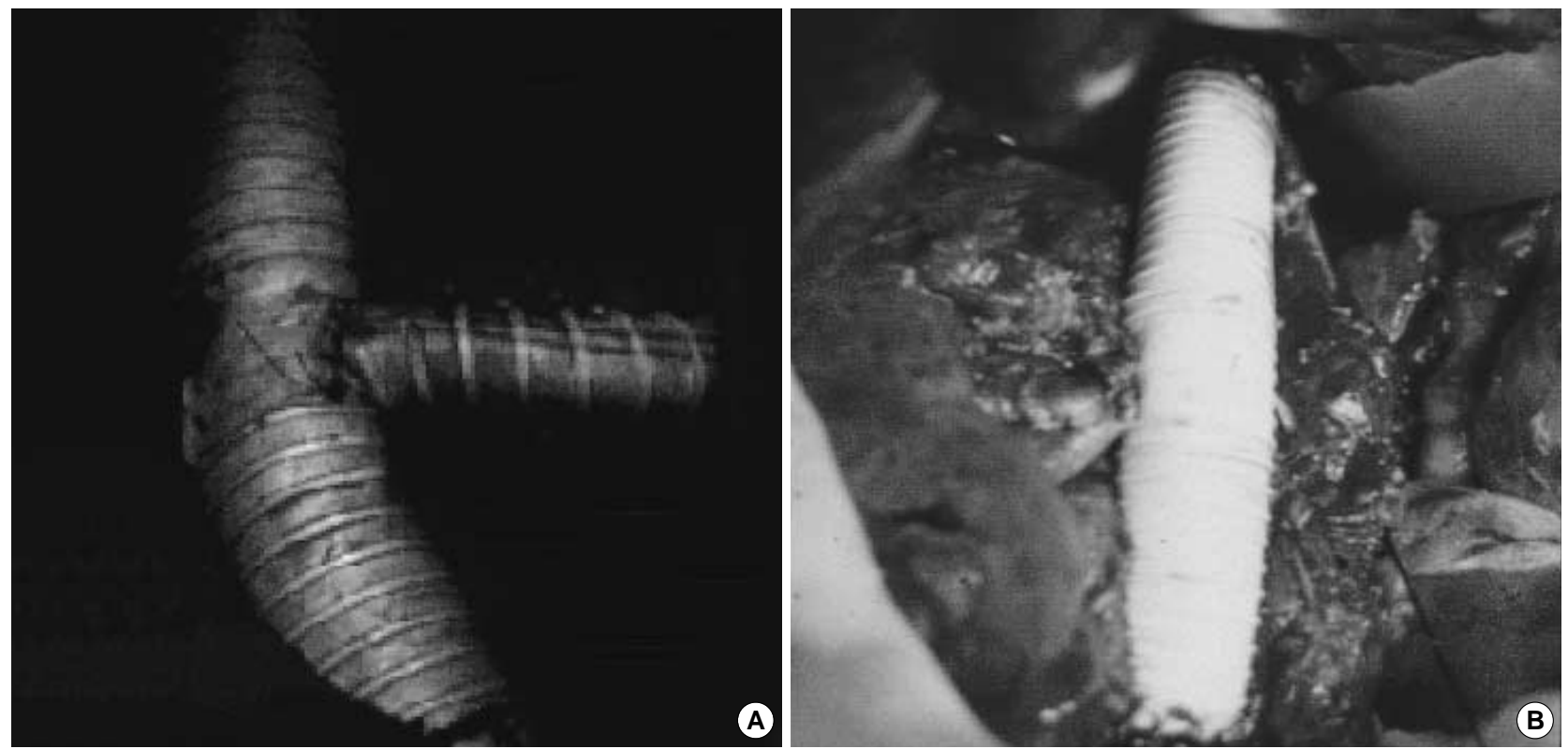

Fig. 2. Operative findings. We routinely performed ringed polytetrafluoroethylene (PTFE) graft interposition and bilateral renal vein reconstructions; direct reimplantation of the right renal vein to the graft (A,B), PTFE graft interposition between the left renal vein and the graft (A), and left renal vein ligation $(B)$, following a complete resection of the tumor.
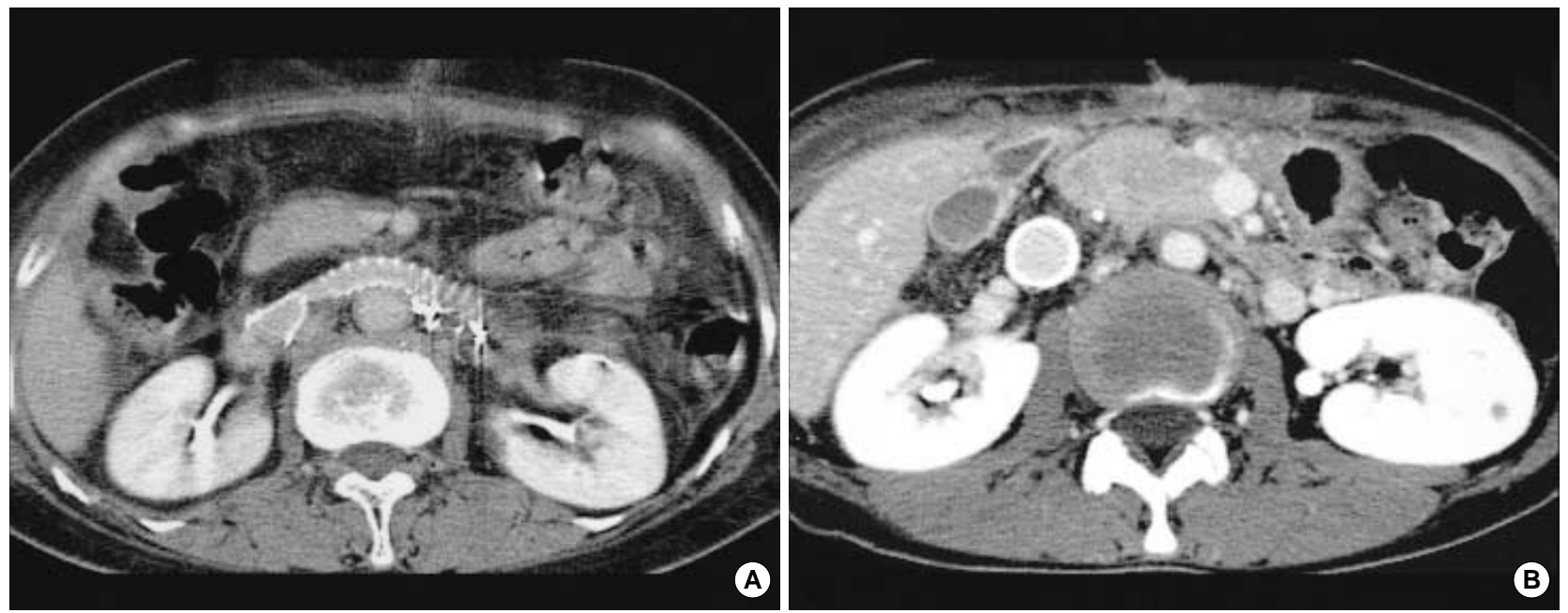

Fig. 3. Postoperative follow-up abdominal computerized tomography scan. The interposed IVC grafts are patent and all kidneys are preserved $(A, B)$. Direct reimplantation of the right renal vein to the graft $(A, B)$ and PTFE graft interposition between the left renal vein and the graft $(A)$ are shown. The left renal vein is not shown in $(B)$, because of ligation during the operation.

masses. All tumors showed a smooth muscle differentiation with interlacing fascicles and positive immunoreaction of smooth muscle actin and desmin. Three tumors showed a well differentiated histology and the remainder was a poorly differentiated tumor.

\section{Recurrence}

During 6 to 41 months follow-up period, a tumor recurrence was noted in one patient in the lung at 18 months after surgery, who was 27 yr old. The tumor showed a poorly differentiated type on pathology, and the patient had no adjuvant radiotherapy.

Table 1 shows the clinical findings and outcomes of all four patients.

\section{DISCUSSION}

Vascular leiomyosarcoma constitutes $2 \%$ of all leiomyosar- 
Table 1. Clinical findings and outcomes of four patients with pararenal leiomyosarcoma of the IVC

\begin{tabular}{|c|c|c|c|c|c|}
\hline \multicolumn{2}{|l|}{ Patient } & 1 & 2 & 3 & 4 \\
\hline \multicolumn{2}{|l|}{ Sex } & $\mathrm{F}$ & $\mathrm{F}$ & $\mathrm{F}$ & $\mathrm{F}$ \\
\hline \multicolumn{2}{|l|}{ Age (yr) } & 56 & 27 & 63 & 57 \\
\hline \multicolumn{6}{|l|}{ Symptom } \\
\hline \multicolumn{2}{|c|}{ Abdominal. Pain } & + & + & + & + \\
\hline \multicolumn{2}{|c|}{ Mass } & - & + & - & - \\
\hline \multirow[t]{2}{*}{ Diagnosis } & \multicolumn{3}{|c|}{$\begin{array}{l}\text { CT scan CT scan } \\
\text { Transfemoral Laparotomy }\end{array}$} & CT scan & CT scan \\
\hline & Tra & \multicolumn{2}{|c|}{$\begin{array}{cc}\text { caval } & \text { and biopsy } \\
\text { biopsy } & \text { (other hospital) }\end{array}$} & & \\
\hline \multirow[t]{2}{*}{$\begin{array}{l}\text { Operation in } \\
4 \text { patients }\end{array}$} & $\begin{array}{l}\text { Resect } \\
\text { PTFE g } \\
\text { Direct r } \\
\text { Left ren }\end{array}$ & $\begin{array}{l}\text { ction of the th } \\
\text { graft interpo } \\
\text { reimplantati } \\
\text { enal vein }\end{array}$ & $\begin{array}{l}\text { umor, } \\
\text { sition ( } 16 \mathrm{~m} \\
\text { on of the rig }\end{array}$ & $\begin{array}{l}\text { gh, ringed), } \\
\text { ght renal vein t }\end{array}$ & to the graft, and \\
\hline & \multicolumn{2}{|c|}{ reconstruction } & \multicolumn{2}{|c|}{ ligation reconstruction } & reconstruction \\
\hline $\begin{array}{l}\text { Operative } \\
\text { Complication }\end{array}$ & & $\begin{array}{l}\text { leeding that } \\
\text { required } \\
\text { a second } \\
\text { aparotomy } \\
\text { for control }\end{array}$ & None & None & None \\
\hline \multicolumn{6}{|l|}{ Findings } \\
\hline \multicolumn{2}{|c|}{ Mass size $(\mathrm{cm})$} & $\begin{array}{c}7.3 \times 4.0 \\
\times 3.5\end{array}$ & $\begin{array}{c}8.9 \times 4.5 \\
\times 3.8\end{array}$ & $\begin{array}{c}8 \times 6.8 \\
\times 4.3\end{array}$ & $\begin{array}{c}5 \times .2 .5 \\
\times 2\end{array}$ \\
\hline Growth pattern Int & Margin involvement & $\begin{array}{c}\text { ntraluminal } \\
(-)\end{array}$ & $\begin{array}{c}\text { Dumbell } \\
(-)\end{array}$ & $\begin{array}{c}\text { Dumbell } \\
(-)\end{array}$ & $\begin{array}{c}\text { Dumbell } \\
(+)\end{array}$ \\
\hline \multicolumn{2}{|l|}{ Cellurality } & ++ & ++ & +++ & ++ \\
\hline \multicolumn{2}{|c|}{ Pleomorphism } & + & +++ & + & ++ \\
\hline \multicolumn{2}{|l|}{ Necrosis (\%) } & 50 & 10 & 30 & 20 \\
\hline \multicolumn{2}{|c|}{ Mitosis (No. of HPF*s } & *s) 10 & 7 & 20 & 5 \\
\hline \multicolumn{6}{|c|}{ Adjuvant therapy } \\
\hline \multicolumn{2}{|c|}{ Chemotherapy } & yes & yes & no & no \\
\hline \multicolumn{2}{|c|}{ Radiotherapy } & yes & no & yes & no \\
\hline \multicolumn{6}{|c|}{ Recurrence } \\
\hline \multicolumn{2}{|l|}{ Local } & no & no & no & no \\
\hline \multicolumn{2}{|l|}{ Systemic } & no & yes & no & no \\
\hline \multicolumn{2}{|c|}{ F/U period (months) } & 41 & 37 & 11 & 6 \\
\hline \multicolumn{2}{|l|}{ Survival } & yes & yes & yes & yes \\
\hline
\end{tabular}

+; mild, ++; moderate, +++; marked, HPF* ; high power field.

comas and involves veins five times more than arteries. More than $50 \%$ of this tumor occurs in the inferior vena cava (3). The origin of the tumor from the IVC is described in relation to the hepatic and renal veins. For this purpose, the IVC is divided into three portions: the upper portion which extends from the entry of the hepatic vein up to the right atrium, the middle portion from the renal vein to the hepatic vein, and the lower portion below the renal vein. However, some tumors cannot be assigned to one portion of the IVC because they originate from the area of the hepatic or renal veins (4).

A complete surgical resection is the only proven therapeutic modality that prolongs the survival in patients with leiomyosarcoma of the IVC. Reconstruction of the IVC is not always required because gradual occlusion of the IVC allows venous collaterals to develop. However, a complete mobilization of the liver from the IVC and preservation of bilateral kidneys are challenging to vascular surgeons at the time of surgery for pararenal location of the tumor. Transient or permanent renal dysfunction may occur in almost one half of patients in whom the suprarenal IVC is resected without vena cava or renal vein reconstruction $(5,6)$. Anuria during operation and/or renal vein pressure above $40 \mathrm{mmHg}$ are indications for the renal vein reconstruction and reimplantation (5). According to the review article of Kulayat et al. (4), ligation of the IVC following segmental resection was commonly performed and graft replacement of the intrahepatic IVC was performed in 11 out of 41 cases. In this situation, the right kidney was removed in all the mentioned cases except one, which was preserved by pelvic autotransplantation of the right kidney. The left kidney was usually preserved by either left renal vein ligation or reimplantation. In our experience, the kidneys were preserved in all patients. Full mobilization of the right and caudate lobes of the liver was the point to perform the curative resection. This procedure resulted in complete exposure of the retrohepatic portion of the IVC and right renal vein, and afforded preservation of the right kidney. Reimplantation of the right renal vein directly to the graft was performed in all patients without any difficulty. Reconstruction of the left renal vein depended on the presence of collaterals with other veins, such as ovarian and adrenal veins. We performed reconstruction of the left renal vein in three patients and ligation of the left renal vein in one patient.

In some cases, venovenous bypass and extracorporeal circulation techniques were used to resect pararenal tumors $(7,8)$. In our experience, a gradual clamping of the proximal IVC under careful monitoring the systemic blood pressure was the way to perform the procedure without venovenous bypass. There was no systemic hypotension or circulatory collapse during the procedure in our cases. Some degree of renal congestion, especially in the right kidney, was noted intraoperatively but relieved on declamping in all cases. As a result, all kidneys were preserved and no postoperative renal functional impairment was noted.

Leiomyosarcoma of the IVC is reported to have a poor prognosis. Over half of patients ( $52.4 \%$ ) who underwent a radical resection had recurred at the median follow-up of 25 months $(2,9)$. Survival of patients with leiomyosarcoma of the IVC depends on the curative resection of tumor and its anatomical site. Involvement of the upper IVC segment and a highgrade tumor had a poor outcome whereas the middle segment tumor was associated with a better outcome $(2,9,10)$. In our study, all four patients were considered to receive a curative resection intraoperatively but one patient showed a positive resection margin on pathologic examination. Histologic grades included three cases of well differentiated tumor and one case of poorly differentiated tumor. We performed adjuvant radiotherapy at a does of 45-55 Gy to all patients except for one patient. Postoperative chemotherapy was given to two patients. Recurrence was noted in one patient in the lung at 18 months after surgery, who showed a poorly differentiated type of the tumor and received no radiotherapy. A controver- 
sy exists in postoperative adjuvant therapy. Although we do not consider the postoperative adjuvant therapy routinely, a postoperative adjuvant radiation therapy seems to be beneficial for the control of the disease. In conclusions, leiomyosarcoma of the inferior vena cava is a rare malignant tumor, where a radical resection is imperative for securing a good prognosis. As for the pararenal leiomyosarcoma of the IVC, full mobilization of the right and caudate lobes of the liver is the key point to perform the curative resection of the tumor and preservation of the right kidney, and a gradual clamping of the proximal IVC under the monitoring the systemic blood pressure is the way to perform the procedure without venovenous bypass. Postoperative adjuvant radiation therapy seems beneficial to improve the survival of the patients with pararenal leiomyosarcoma of the IVC.

\section{REFERENCES}

1. Dzsinich C, Gloviczki P, van Heerden JA, Nagorney DM, Pairolero PC, Johnson CM, Hallett JW Jr, Bower TC, Cherry KJ Jr. Primary venous leiomyosarcoma: a rare but lethal disease. J Vasc Surg 1992; 15: 595-603.

2. Mingoli A, Feldhaus RJ, Cavallaro A, Stipa S. Leiomyosarcoma of the inferior vena cava: Analysis and search of world literature on 141 patients and report of three new cases. J Vasc Surg 1991; 14: 688-99.
3. Kevorkian J, Cento DP. Leiomyosarcoma of large arteries and veins. Surgery 1973; 73: 390-400.

4. Kulaylat MN, Karakousis CP, Doerr RJ, Karamanoukian HL, O 'Brien J, Peer R. Leiomyosarcoma of the inferior vena cava: A clinicopathologic review and report of three cases. J Surg Oncol 1997; 65: 205-17.

5. Huguet C, Ferry M, Gavelli A. Resection of the suprarenal inferior vena cava: the role of prosthetic replacement. Arch Surg 1995; 130: 793-7.

6. Bower TC, Stanson AW. Tumors of the inferior vena cava: diagnosis and management. In Rutherford RB, ed: Vascular surgery, 5th ed, Philadelphia, 2000, WB Saunders.

7. Monig SP, Gawenda M, Erasmi H, Zieren J, Pichlmaier H. Diagnosis, treatment and prognosis of leiomyosarcoma of the inferior vena cava. Three cases and summary of published reports. Eur J Surg 1995; 161: $231-5$.

8. Yanaga K, Okadome K, Ito H, Matsumata T, Makino T, Okamura H, Sugimachi K. Graft replacement of pararenal inferior vena cava for leiomyosarcoma with the use of venovenous bypass. Surgery 1993; 113: 109-12

9. Mingoli A, Cavallaro A, Sapienza P, Di Marzo L, Feldhaus RJ, Cavallari $\mathrm{N}$. International registry of inferior vena cava leiomyosarcoma: analysis of a world series on 218 patients. Anticancer Res 1996; 16: 3201-5.

10. Burke AP, Virmani R. Sarcomas of the great vessels. A clinicopathologic study. Cancer 1993; 71: 1761-73. 